\title{
Cortical Hypoexcitability in Chronic Smokers? A Transcranial Magnetic Stimulation Study
}

\author{
Nicolas Lang*,', ${ }^{*}$ Alkomiet Hasan', Elke Sueske', Walter Paulus' and Michael A Nitsche' \\ 'Department of Clinical Neurophysiology, Georg-August University, Göttingen, Germany; ${ }^{2}$ Department of Neurology, Christian-Albrechts \\ University, Kiel, Germany
}

\begin{abstract}
Studies in animal models and humans indicate that chronic nicotine intake influences neuronal excitability, resulting in functional and structural CNS changes. The aim of the present study was to explore human primary motor cortex (MI) excitability with transcranial magnetic stimulation (TMS) in chronic smokers. A total of 44 right-handed volunteers, aged 20-30 years, participated in the study. Chronic smokers were compared with age- and sex-matched healthy nonsmokers. We tested cortical excitability with single- and pairedpulse TMS to the left MI and short-latency afferent inhibition (SAI) by combining median nerve stimulation and motor cortex TMS. Compared with nonsmoking controls, chronic smokers showed a significantly larger amount of SAl, which is thought to depend upon the activity of cholinergic inhibitory circuits produced by somatosensory inputs. Moreover, TMS-evoked inhibitory cortical silent periods were prolonged, whereas paired-pulse intracortical facilitation and motor-evoked potentials during moderate contraction were reduced. The results suggest that chronic nicotine intake may not only strengthen cholinergic inhibitory circuits, but could also be associated with enhanced inhibitory and reduced facilitatory mechanism of specific neuronal circuits in motor cortex. These changes may form a physiological basis for neurobiological and behavioral variations associated with chronic smoking.

Neuropsychopharmacology (2008) 33, 25 I7-2523; doi:I 0. I038/sj.npp. I 301645; published online 5 December 2007
\end{abstract}

Keywords: smoking; nicotine; motor cortex; TMS; intracortical facilitation; afferent inhibition

\section{INTRODUCTION}

Nicotine is the main neuroactive component of tobacco, and thus might explain some of the addictive features of tobacco consumption. However, knowledge about the specific effect of nicotine on the central nervous system is far from being complete. The functional impact of nicotine has been studied in humans and animals to some extent. On a cognitive level, acute and chronic exposure to nicotine has been shown to improve attention and working memory (Hahn and Stolerman, 2002; Kumari et al, 2003; Thiel et al, 2005). On the level of neuropsychiatric diseases, such as schizophrenia, nicotine might be used by smokers to compensate for subtle cognitive deficits. It has been shown that smokers display reduced working and verbal memory capacity compared to nonsmokers, especially under nicotine withdrawal (Jacobsen et al, 2005). Similarly, schizophrenic patients exhibit visuospatial working memory deficits under nicotine abstinence, which is improved relevantly by nicotine consumption (Sacco et al, 2005).

\footnotetext{
*Correspondence: Dr N Lang, Department of Neurology, ChristianAlbrechts University, Schittenhelmstr. 10, Kiel 24105, Germany, Tel: + 4943 I 5978709 , Fax: + 494315978502 ,

E-mail: n.lang@neurologie.uni-kiel.de

Received 4 October 2007; revised 30 October 2007; accepted 31 October 2007
}

Knowledge about the neurophysiological foundation of these effects of nicotine, however, is largely restricted to animal experimentation. It is known from in vitro animal experiments that the nicotinic acetylcholine receptor (nAChR) induces neuronal depolarization by a transmembrane cationic inward current. Hereby, calcium seems to play a dominant role (Burnashev, 1998). Since most of the cholinergic neurons do not terminate on postsynaptic sites, acetylcholine and nicotine have been characterized more as neuromodulators than transmitters (Mansvelder et al, 2006). nAChR modulate glutamate, dopamine, and GABA release primarily presynaptically. Nicotine also activates presynaptic non-a7 nAChRs on feed-forward interneurons and hereby decreases the evoked release of GABA onto pyramidal cells (Yamazaki et al, 2005). Furthermore, nicotine increases the frequency of spontaneous inhibitory postsynaptic currents (IPSCs) in pyramidal cells, and concomitantly causes a reduction in the size of responses to focal GABA application onto pyramidal cells, suggesting that the nicotine-induced increase in interneuronal activity leads ultimately to a use-dependent depression of evoked IPSCs in pyramidal cells (Yamazaki et al, 2005). Consequently activation of nicotine receptors can result in complex interactions involving inhibition or disinhibition of pyramidal cortical neurons (Alkondon et al, 2000), and the translation into functional effects is not easily done. The situation is complicated further by the fact that chronic 
exposition to nicotine induces long-lasting modifications of cortical architecture, like dendritic arborization and nicotinic receptor density (Breese et al, 1997; Gonzalez et al, 2005).

The neurophysiological action of nicotine in humans is largely unknown so far. In accordance with animal experiments, enhancement of acetylcholine receptor activity by a single oral dose of the acetylcholinesterase inhibitor tacrine has been shown to decrease intracortical inhibition and increase facilitation in healthy subjects (Korchounov et al, 2005). However, in Alzheimer's patients, who show reduced intracortical inhibition compared to age-matched controls, treatment with the cholinesterase inhibitor donepezil increased intracortical inhibition (Liepert et al, 2001a). The relative contribution of nicotinic receptors to these effects was explored in only one study, where it was demonstrated that reduced intracortical inhibition and short interval afferent inhibition was normalized in patients with Tourette's syndrome after a single dose of nicotine (Orth et al, 2005).

To shed some more light on the impact of nicotine on cortical function in humans, we explored the influence of chronic nicotine consumption on motor cortical network excitability by comparing age- and sex-matched groups of otherwise healthy smokers and nonsmokers with transcranial magnetic stimulation (TMS). TMS gives the possibility of exploring noninvasively the mode of action of neuroactive substances in the intact human brain, and allows qualitative and quantitative evaluation of distinct neuronal networks, which might be involved in the action of nicotine. Our hypothesis was that chronic smokers would show abnormal excitability of neuronal circuits involving motor cortex compared to controls, predominant in those circuits with considerable influence of cholinergic receptor activity.

\section{MATERIALS AND METHODS}

\section{Subjects}

Altogether 44 university students (age range 20-30 years) participated in the study. General exclusion criteria were cardiac pacemaker; metal implants in the head; age younger than 18 or older than 45 years; current intake of any medication; current or previous neurological, psychiatric, or internal diseases; pregnancy or breastfeeding; current or previous drug (other than nicotine) or alcohol abuse; or participation in another clinical trial within the last 8 weeks. Chronic smokers had to be continuous and uninterrupted consumers of at least 10 cigarettes per day within the past 4 years, with a score in the Fagerstrom Test for Nicotine Dependence of 1-5 indicating mild levels of nicotine dependence (Heatherton et al, 1991). In order to minimize acute nicotine effects immediately after consumption as well as withdrawal effects, smokers were always ask to smoke their last cigarette $1 \mathrm{~h}$ before the beginning of the individual experimental session. Nonsmokers had to be without a history of continuous smoking and without occasional nicotine consumption within the past 4 years. All subjects were right-handed, according to the Edinburgh Handedness Inventory (Oldfield, 1971). The study was conducted according to the Declaration of Helsinki and approval was obtained from the Ethics Committee of the Georg-August University, Göttingen. All subjects gave their informed written consent before participation.

\section{Data Acquisition}

During the experiments subjects were comfortably seated in a reclining chair with head and arm rests. Surface electromyogram (EMG) was recorded from a right hand muscle through a pair of $\mathrm{Ag}-\mathrm{AgCl}$ surface electrodes in a belly-tendon montage. Raw signals were amplified, bandpass filtered $(3 \mathrm{~Hz}-3 \mathrm{kHz})$, digitized with a micro $1401 \mathrm{AD}$ converter (Cambridge Electronic Design, Cambridge, UK) controlled by Signal Software (Cambridge Electronic Design, version 2.13), and stored on a personal computer for offline analysis. Complete relaxation was controlled through auditory and visual feedback of EMG activity. TMS was performed by using a Magstim standard double ('figureof-eight') 70-mm coil connected to a monophasic Magstim 200 stimulator (experiment 1) or to two Magstim 200 stimulators via a bistim module (experiment 2) (all TMS devices manufactured by the Magstim Company, Dyfed, UK). The coil was held tangentially to the skull over the left primary motor cortex (M1) with the handle pointing posterolaterally at a $45^{\circ}$ angle to the sagittal plane. This orientation of the induced electrical field is thought to be optimal for a predominantly transsynaptic mode of activation of the corticospinal system (Di Lazzaro et al, 1998). At the beginning of each session, the optimal position of the TMS coil over the left M1 for eliciting MEP in the resting hand muscle was assessed. The site was marked with a skin marker to ensure that the coil was held in the correct position throughout the experiment.

\section{Experiment 1 (Afferent Inhibition)}

A total of 24 subjects participated in the first experiment. This experiment was performed in order to examine SAI and long-latency afferent inhibition (LAI), which can be determined by a conditioning-test paradigm of combined peripheral and cortical stimulation (Di Lazzaro et al, 2000; Tokimura et al, 2000). Participants for this experiment were divided into two age- and sex-matched groups of 12 smokers ( 10 men, 2 women; mean age $25 \pm 0.57$ years) and 12 nonsmoking controls (10 men, 2 women; mean age $24 \pm 0.7$ years). Conditioning stimuli were single electrical pulses generated by a Digitimer D185 stimulator (Digitimer Ltd., Welwyn Garden City, UK) applied through bipolar electrodes to the right median nerve at the wrist. Compared to previous studies (Di Lazzaro et al, 2000; Tokimura et al, 2000 ), a relatively low intensity of the conditioning stimulus ( $80 \%$ of the individual electric motor threshold, EMT, of the nerve) was chosen in order to avoid an influence from muscle contractions by the conditioning stimulus. The intensity of the cortical (test) stimulus was adjusted to elicit MEP in the relaxed APB with peak-to-peak amplitudes of approximately $1 \mathrm{mV}$. The conditioning stimulus to the peripheral nerve preceded the magnetic test stimulus by interstimulus intervals (ISIs) of 20,40,100, and $200 \mathrm{~ms}$. An ISI of $20 \mathrm{~ms}$ can be used to study SAI, whereas 100 and $200 \mathrm{~ms}$ will lead to LAI. In random order the control condition (test pulse alone) was tested 30 times and each of 
the conditioning-test pairs 15 times. The amplitude of the conditioned MEP was expressed as a percentage of the amplitude of the test MEP.

\section{Experiment 2 (Motor Cortex Excitability)}

A total of 38 subjects ( 18 of whom also took part in the first experiment) participated in the second experiment, which was performed in order to examine a variety of other TMS measurements describing inhibitory and facilitatory circuits in M1. Participants were again divided into two age- and sex-matched groups, now consisting of 19 smokers (13 men, 6 women; mean age $24 \pm 0.44$ years) and 19 nonsmoking controls ( 13 men, 6 women; mean age $24 \pm 0.35$ years).

TMS measurements included resting motor threshold (RMT) and active motor threshold (AMT), the TMS-intensity to evoke MEP of approximately 1-mV peak-to-peak amplitude (SI1mV), I/O curves, SICI/ICF, LICI, active MEP (aMEP) during moderate tonic activation, and the cortical silent period (CSP). Stimulus intensities (in percentage of maximal stimulator output) were determined at the beginning of each experiment, starting with SI1mV. RMT was defined as the minimal output of the stimulator that induced a reliable MEP (approximately $50 \mu \mathrm{V}$ in amplitude) in at least 5 of 10 consecutive trials in the relaxed FDI, and AMT was defined as the lowest stimulus intensity at which 5 of 10 consecutive stimuli elicited reliable MEP (approximately $200 \mu \mathrm{V}$ in amplitude) in the tonically contracted FDI (Rothwell et al, 1999). I/O curves were measured with three different stimulus intensities (100, 120, and $140 \%$ RMT), each with 10 pulses. A mean was calculated for each intensity. SICI/ICF and LICI were measured with different protocols of single- and paired-pulse TMS applied at $0.25 \mathrm{~Hz}$. For SICI/ICF, two magnetic stimuli were given through the same stimulating coil, and the effect of the first (conditioning) stimulus on the second (test) stimulus was investigated (Kujirai et al, 1993). To avoid floor or ceiling effects, the intensity of the conditioning stimulus was set to $90 \%$ AMT. The test-stimulus intensity was adjusted to SI $1 \mathrm{mV}$. SICI/ICF was measured with ISIs of 2, 4, 7, 9, and $12 \mathrm{~ms}$. The control condition (test pulse alone) was tested 40 times, each of the conditioning-test pairs 20 times, and conditions were applied in randomized order. The mean amplitude of the conditioned MEP at each ISI was expressed as a percentage of the mean size of the unconditioned test pulse. SICI was taken as the mean percentage inhibition at ISIs of 2 and $4 \mathrm{~ms}$, whereas ICF was taken as the mean facilitation at ISIs of 9 and $12 \mathrm{~ms}$. Another protocol tested LICI with two suprathreshold stimuli applied with ISIs of 50,100 , and $150 \mathrm{~ms}$ (Valls-Sole et al, 1992). The intensity of both stimuli was set to the relatively low value of $110 \%$ RMT to avoid floor effects. The control condition (first pulse alone) was tested 30 times, each of the paired stimuli 15 times, and conditions were applied in randomized order. LICI was taken as the mean percentage inhibition of conditioned MEP at ISIs of 50, 100, and $150 \mathrm{~ms}$. At the end of each session, 10 pulses with SI $1 \mathrm{mV}$ and 120\% RMT, respectively, were applied during moderate tonic contraction of the FDI (approximately $30-50 \%$ of maximal voluntary contraction). The mean peak-to-peak MEP amplitude (in $\mathrm{mV}$ ) was taken from each intensity for aMEP. CSPs were separately determined in rectified and averaged EMG traces with a prestimulus period of $100 \mathrm{~ms}$. CSP (in ms) was measured from the TMS stimulus artifact to the point where the signal reached the amplitude of the mean prestimulus EMG activity again for $>5 \mathrm{~ms}$.

\section{Data Analyses}

For both experiments and each measure (SAI/LAI, SI1mV, RMT, AMT, I/O curve, SICI/ICF, LICI, aMEP, and CSP), we performed separate analyses of variance (ANOVAs) for repeated measurements by using mean values from each subject as the dependent variable. In addition to the factor 'group' (smokers $v s$ nonsmokers), the ANOVA model included the factor 'ISI' $(20,40,100$, and $200 \mathrm{~ms})$ when SAI/LAI was analyzed, or 'ISI' $(2,4,7,9$, and $12 \mathrm{~ms}$ ) when SICI/ICF was analyzed, or the factor 'intensity' $(100,120$, and 140\% RMT) for I/O curves, or 'intensity' (120\% RMT and SI $1 \mathrm{mV}$ ) for CSP and aMEP. Paired-samples two-tailed $t$-tests were used to compare thresholds (EMT, RMT, AMT) and TMS intensities (SI1mV) between groups in both experiments. The Greenhouse-Geisser method was used when necessary to correct for nonsphericity. Conditional on a significant F-value in ANOVA, paired-samples two-tailed $t$-tests were used for post hoc analysis, and a $p$-value of $<0.05$ was considered significant for all statistical analyses. Data are expressed as mean \pm SEM.

\section{RESULTS}

\section{Fagerstrom Scores}

Mean Fagerstrom score of the smoking groups were $2.92 \pm$ 0.4 in the first experiment (afferent inhibition) and $3.19 \pm$ 0.24 in the second experiment (motor cortex excitability). This indicates low levels of nicotine dependence in both experiments.

\section{Stimulation Parameters}

Mean stimulation parameters from both experiments are given in Table 1. Paired-samples $t$-tests did not detect significant differences for peripheral nerve and cortex stimulation parameters between groups (experiment 1: EMT $t=$ $0.369, \mathrm{df}=11, p=0.536 ;$ and SI $1 \mathrm{mV} t=0.421, \mathrm{df}=11$,

Table I Mean Values of Electric Motor Threshold, Stimulus Intensity Necessary to Evoke MEP of I-mV Amplitude, Resting Motor Threshold, and Active Motor Threshold from Both Experiments

\begin{tabular}{|c|c|c|c|c|c|}
\hline & \multicolumn{2}{|c|}{ Experiment I } & \multicolumn{3}{|c|}{ Experiment 2} \\
\hline & $\mathrm{EMT}^{\mathrm{a}}$ & SIImVb & $\mathbf{R M T} \mathbf{T}^{\mathbf{b}}$ & $A M T^{b}$ & SIImV \\
\hline Smokers & $144 \pm 19$ & $45 \pm 3$ & $51 \pm 2$ & $37 \pm 1$ & $63 \pm 2$ \\
\hline Nonsmokers & $153 \pm 13$ & $48 \pm 3$ & $50 \pm 1$ & $37 \pm 1$ & $61 \pm 2$ \\
\hline
\end{tabular}

Abbreviations: AMT, active motor threshold; EMT, electric motor threshold; RMT, resting motor threshold; SII mV, I-mV peak-to-peak amplitude.

Paired-samples $t$-test did not reveal significant differences between groups (smokers vs nonsmokers) for any of these parameters. ( ${ }^{a}$ in volts; ${ }^{b}$ in $\%$ of maximal stimulator output). 
$p=0.682$; experiment 2 : RMT $t=0.316, \mathrm{df}=18, p=0.756$; AMT $t=0.306, \mathrm{df}=18, p=0.764 ;$ and SI $1 \mathrm{mV} t=0.558$, $\mathrm{df}=18, p=0.583$ ).

\section{Afferent Inhibition}

Smokers showed significantly more SAI compared to nonsmoking controls, whereas LAI did not differ between groups (Figure 1). ANOVA on mean SAI/LAI values demonstrated a significant main effect for the factor 'ISI' $(F(3,33)=14.6$, $p<0.001)$ and a significant interaction 'group' by 'ISI' $(\mathrm{F}(3,33)=3.2, p=0.038)$. Post hoc analysis revealed a significant difference between groups at ISI $20 \mathrm{~ms}$, indicating that SAI at this interval was more pronounced in the smokers group compared to nonsmokers (post hoc $t$-test: $t=4.093$, $\mathrm{df}=11, p=0.002)$.

\section{Motor Cortex Excitability}

At rest, smokers demonstrated reduced ICF compared to nonsmokers (Figure 2). ANOVA on SICI/ICF showed a trend for the factor 'group' $(\mathrm{F}(1,18)=3.6, p=0.074)$, a significant

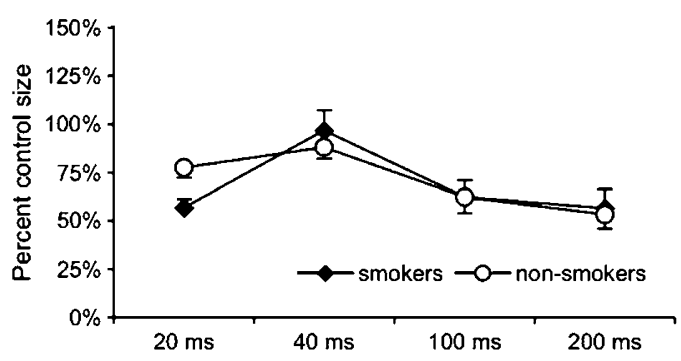

Figure I Increased short-latency afferent inhibition in smokers. Afferent inhibition was tested at rest by combining median nerve stimulation and motor cortex TMS with interstimulus intervals of $20 \mathrm{~ms}$ (short-latency afferent inhibition), $40 \mathrm{~ms}$ (indifferent interval), as well as 100 and $200 \mathrm{~ms}$ (long-latency afferent inhibition). Amplitudes of the conditioned MEP are expressed as a percentage of the amplitudes of the test MEP (TMS alone). Data are represented as mean \pm SEM. SAI at 20 ms was found significantly more pronounced in smokers compared to nonsmokers (ANOVA, post hoc $t$-test; for details see Results), whereas LAI did not differ between groups.

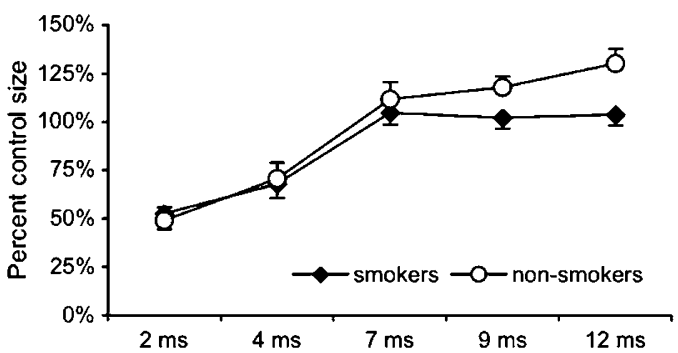

Figure 2 Reduction of intracortical facilitation in smokers. Short-latency intracortical inhibition and facilitation was tested at rest with paired-pulse motor cortex TMS at interstimulus intervals of $2-12 \mathrm{~ms}$. Amplitudes of the conditioned MEP are expressed as a percentage of the amplitudes of the test MEP (single TMS pulse). Data are represented as mean \pm SEM. Intracortical facilitation at interstimulus intervals 9 and 12 ms was found significantly reduced in smokers compared to nonsmokers (ANOVA, post hoc $t$-test; for details see Results), whereas short-latency intracortical inhibition did not differ between groups. effect for the factor 'ISI' $(\mathrm{F}(3,60)=52.1, p<0.001)$, and a significant interaction 'group' by 'ISI' $(\mathrm{F}(3,55)=3.3, p=$ 0.025). Post hoc analyses demonstrated that smokers had significantly less ICF at ISI $9 \mathrm{~ms}(t=-2.289, \mathrm{df}=18, t=$ $0.034)$ and $12 \mathrm{~ms}(t=-3.272, \mathrm{df}=18, p=0.004)$ compared with nonsmokers. Thus, mean ICF, expressed as an arithmetic mean of ICF at 9 and $12 \mathrm{~ms}$, was significantly reduced in smokers $(t=-2.924, \mathrm{df}=18, p=0.009)$. Figure 3 illustrates data from parameters obtained during voluntary activation of the motor system (CSP and aMEP). ANOVA on CSP showed a significant main effect for the factor 'group' $(\mathrm{F}(1,18)=4.8, p=0.042)$ and for 'intensity' $(\mathrm{F}(1,18)=7.7$, $p=0.013)$, but not for an interaction of the two factors. This indicates differences in CSP between groups as well as between intensities, and that differences in CSP between groups were independent of intensities used to evoke CSP. Differences between intensities can be explained by a wellknown positive correlation of CSP duration with stimulus intensity (Haug et al, 1992). Post hoc $t$-tests demonstrated a significant prolongation of CSP in smokers compared to nonsmokers when SI1mV was used as intensity $(t=2.402$, $\mathrm{df}=18, p=0.027)$ and a trend with $120 \% \operatorname{RMT}(t=1.858$, $\mathrm{df}=18, p=0.082)$. ANOVA on aMEP revealed a significant main effect for the factor 'group' $(\mathrm{F}(1,18)=5.9, p=0.026)$, but not for the factor 'intensity' and no interaction of the two factors. This indicates that differences in aMEP between groups were independent of intensities used to evoke aMEP. Post hoc $t$-tests demonstrated a significant reduction of aMEP in smokers compared to nonsmokers when SI1 mV was used as intensity $(t=-2.052, \mathrm{df}=18, p=0.015)$ and a trend with $120 \%$ RMT $(t=-2.687, \mathrm{df}=18, p=0.055)$. Apart from an inherent effect for 'intensity' on I/O curve values $(\mathrm{F}(2,36)=67.6, p<0.001)$ separate ANOVAs on LICI and
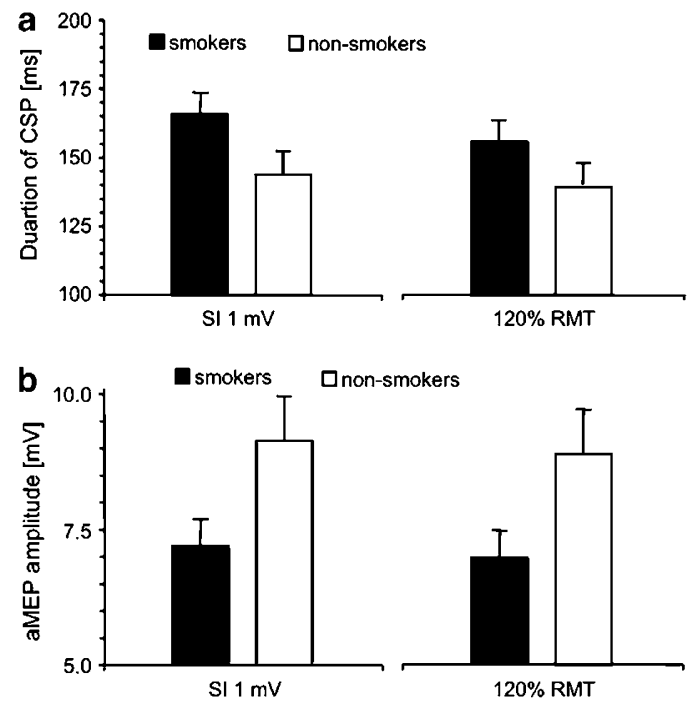

Figure 3 Increased inhibition and reduced facilitation during motor system activation in smokers. Cortical silent periods (CSP, a) and active MEP (aMEP, b) were evoked with two different stimulus intensities (I-mV peak-to-peak amplitude (SIImV) and 120\% resting motor threshold (RMT)) during moderate contraction of the target muscle. Data are represented as mean \pm SEM. The duration of CSP was found significantly prolonged and aMEP amplitude reduced in smokers compared to nonsmokers, irrespective of the mode of measure (ANOVA, post hoc t-test, for details see Results). 
I/O curve values revealed no significant main effects or interactions, and motor thresholds or stimulation intensities did not differ between groups (Table 1).

\section{DISCUSSION}

The present study investigated human motor cortex excitability in chronic smokers with TMS, an approach with advantages and limitations. Advantages include the possibility of exploring noninvasively inhibitory and excitatory cortical networks in the intact human under chronic nicotine exposure and comparing the pattern of excitability with those induced by pharmacological agents or found in neuropsychiatric disorders. In contrast to most animal research, TMS can describe alterations at the system level of the human cerebral cortex, often being more closely related to the clinical context. Potential limitations of the present study may be that the observed effects could partially be a reflection of acute nicotine administration rather than the effect of chronic smoking, as well as that the study design did not control completely for a trait that predisposes to nicotine consumption. We attempted to reduce the problem of acute nicotine administration to a minimum by performing the experiments within the second hour after smoking cessation, hence to the latest possible time point before relevant withdrawal symptoms would arise. Personality traits can influence TMS measures of motor cortical excitability, as has been shown ie in anxiety (Wassermann et al, 2001). However, the fact that chronic smokers in our study showed only low nicotine dependence reduces this weakness.

Despite this limitation, the present study supplies novel insights into cerebral cortex physiology of chronic smokers. It demonstrates that chronic smokers differ from nonsmoking individuals with regard to motor-cortical excitability tested with TMS. Specifically, smokers display enhanced short-latency afferent inhibition (SAI), prolonged inhibitory silent periods, reduced intracortical facilitation and lower aMEP amplitudes. This pattern of results argues for specific differences of excitability between both groups on the cortical level. While the above-mentioned protocols test different intracortical neuronal circuits, corticospinal excitability, as tested by the I/O curve, the TMS intensity necessary to elicit 1-mV MEP amplitudes, and motor thresholds were identical between the groups. Also, no difference could be found between groups regarding LAI, which probably involves the basal ganglia-thalamocortical loop (Sailer et al, 2003).

The aMEP amplitude is a global measure of motor-cortical excitability, which is influenced by ion channel-activity, GABAergic, glutamatergic, dopaminergic, adrenergic, and cholinergic receptors (for review see Ziemann, 2003). The reduction of MEP amplitudes in smokers is in clear favor for enhanced motor-cortical inhibition, but the multitude of mechanisms involved, added by the indirect modulatory effects of nicotine on diverse types of receptors does not allow to derive definite conclusions about the specific mechanism how nicotine reduces motor cortex excitability from these results.

However, SAI is known to reflect specifically the inhibitory effect of somatosensory afferents to the M1 (Tokimura et al,
2000). In a patient with paramedian thalamic stroke absence of SAI could be demonstrated on the affected side, suggesting that thalamocortical projections from the paramedian thalamus contribute to the integration of sensory input at the cortical level (Oliviero et al, 2005). The inhibitory action of SAI seems to be cholinergically driven and it was demonstrated that it is reduced by the muscarinic receptor antagonist scopolamine (Di Lazzaro et al, 2000). The results of our study are in favor for an additional influence of nicotine on this neuronal circuit, ie that in smokers nicotine might enhance the somatosensory inhibition of the M1 by activation of cholinergic synapses, which can be due to activation of nicotinic receptors or by the indirect activation of the muscarinic receptors. Another motor-cortical inhibitory mechanism, which is measured by the CSP, reflects a long-lasting intracortical inhibition mediated by $\mathrm{GABA}_{\mathrm{B}}$ receptors (Inghilleri et al, 1996; Siebner et al, 1998). Our results demonstrate that, like SAI, CSP is enhanced in smokers. Since nicotine is known to enhance the expression of $\mathrm{GABA}_{\mathrm{B}}$ receptors ( $\mathrm{Li}$ et al, 2004), this is one likely explanation for the prolongation of CSP our smokers group. Interestingly also intracortical facilitation is, among others, modulated by $\mathrm{GABA}_{\mathrm{B}}$-receptor activity (Ziemann et al, 1996). Its reduction in the smokers group might thus also be due to an enhanced expression of these receptors induced by nicotine.

A possible alternative explanation for the present observations is that nicotine activates cholinergic circuits via muscarinic receptors. This would explain the SAI increase and MEP amplitude reduction, which is the opposite finding of the work by Di Lazzaro et al (2000) with blocking muscarinic receptors. Nicotinic, but not muscarinic receptors could also activate an alternative, non-GABAergic pathway that could explain the CSP prolongation.

However, although these mechanisms of function seem plausible, it should be kept in mind that the TMS protocols tested here are not restricted to the influence of one type of receptor. For example, dopaminergic drugs enhance CSP duration and reduce ICF, and NMDA receptors are also involved in ICF generation (for review see Ziemann, 2004). Given the neuromodulatory action of nicotine on diverse receptor types, alternative mechanisms of action are conceivable. This is underlined by the fact that the $\mathrm{GABA}_{\mathrm{B}}$ agonist baclofen has been described to enhance SICI elsewhere (Ziemann et al, 1996), but SICI was identical for both subject groups in our study. Thus, the hypothesis of an effect of nicotine restricted to cholinergic receptors and $\mathrm{GABA}_{\mathrm{B}}$ activity seems questionable. Future studies should explore this to a larger extent.

Our results are not in full accordance with those of former studies. Enhancement of acetylcholine by a single oral dose of tacrine reduced SICI and enhanced ICF in healthy subjects in another study (Korchounov et al, 2005), and a similar effect was achieved by a single dose of the muscarinic antagonist atropine (Liepert et al, 2001b). Moreover, application of a cholinesterase antagonist in Alzheimer's patients and of nicotine in patients with Tourette's syndrome increased SICI (Orth et al, 2005). This seemingly opposing effects of ACh modulation in different studies underline the fact that it acts as a neuromodulator and thus the direction of its action might critically depend not only on the subreceptors involved, but also on receptor 
availability, baseline activation, chronic $v s$ acute administration, and other factors.

As mentioned above, a possible limitation of this study is that we did not look directly for causality, that is we did not modulate nicotine in our subjects and therefore it cannot be concluded clearly if the cortical excitability differences between the groups under study are caused by nicotine consumption or are representing a kind of proneness for nicotine dependency in the smokers group. The situation is even more complicated by the fact that chronic exposition to nicotine leads to an enhancement and desensitization of nicotinic, but to enhanced affinity of muscarinic receptors to acetylcholine (Breese et al, 1997; Flores et al, 1992; Schwartz and Kellar, 1985; Wang and Sun, 2005) and to structural cortical alterations (Gonzalez et al, 2005). Thus, the complex interplay between inherent cortical excitability differences between smoking and nonsmoking individuals, its modification by chronic nicotine consumption, and the acute effects of nicotine should be taken into account in future studies.

Taken together, the results of the current study show a cortical excitability reduction in smokers as compared to nonsmoking individuals. It might be speculated that this functional cortical modification is due to chronic nicotine consumption. A possible functional relevance of enhanced cortical inhibition might be a reduction of cortical noise, thus enhancing the salience of stimuli-associated information processing. Indeed, acetylcholine has been proposed to increase cortical signal-to-noise ratio and to improve cognitive functioning (Gu, 2003). This mechanism might also be relevant for diseases displaying pathologically reduced inhibition or signal-to-noise ratio, as in Tourette's syndrome or schizophrenia.

\section{ACKNOWLEDGEMENTS}

The study was supported by the Deutsche Forschungsgemeinschaft (DFG grant NI 683/4-1 'Towards risk prediction of nicotine dependency by exploring individual limits of cortical neuroplasticity in humans') within the DFG priority program 'Nicotine: Molecular and Physiological Effects in Central Nervous System'.

\section{DISCLOSURE/CONFLICT OF INTEREST}

The authors declare that, except income received from primary employers, no financial support or compensation has been received from any individual or corporate entity over the past 3 years for research or professional service and there are no personal finance holdings that could be perceived as constituting a potential conflict of interest.

\section{REFERENCES}

Alkondon M, Pereira EF, Almeida LE, Randall WR, Albuquerque EX (2000). Nicotine at concentrations found in cigarette smokers activates and desensitizes nicotinic acetylcholine receptors in CA1 interneurons of rat hippocampus. Neuropharmacology 39: 2726-2739.

Breese CR, Marks MJ, Logel J, Adams CE, Sullivan B, Collins AC et al (1997). Effect of smoking history on [3H]nicotine binding in human postmortem brain. J Pharmacol Exp Ther 282: 7-13.
Burnashev N (1998). Calcium permeability of ligand-gated channels. Cell Calcium 24: 325-332.

Di Lazzaro V, Oliviero A, Profice P, Pennisi MA, Di Giovanni S, Zito $\mathrm{G}$ et al (2000). Muscarinic receptor blockade has differential effects on the excitability of intracortical circuits in the human motor cortex. Exp Brain Res 135: 455-461.

Di Lazzaro V, Oliviero A, Profice P, Saturno E, Pilato F, Insola A et al (1998). Comparison of descending volleys evoked by transcranial magnetic and electric stimulation in conscious humans. Electroencephalogr Clin Neurophysiol 109: 397-401.

Flores CM, Rogers SW, Pabreza LA, Wolfe BB, Kellar KJ (1992). A subtype of nicotinic cholinergic receptor in rat brain is composed of alpha 4 and beta 2 subunits and is up-regulated by chronic nicotine treatment. Mol Pharmacol 41: 31-37.

Gonzalez CL, Gharbawie OA, Whishaw IQ, Kolb B (2005). Nicotine stimulates dendritic arborization in motor cortex and improves concurrent motor skill but impairs subsequent motor learning. Synapse 55: 183-191.

$\mathrm{Gu} \mathrm{Q}$ (2003). Contribution of acetylcholine to visual cortex plasticity. Neurobiol Learn Mem 80: 291-301.

Hahn B, Stolerman IP (2002). Nicotine-induced attentional enhancement in rats: effects of chronic exposure to nicotine. Neuropsychopharmacology 27: 712-722.

Haug BA, Schonle PW, Knobloch C, Kohne M (1992). Silent period measurement revives as a valuable diagnostic tool with transcranial magnetic stimulation. Electroencephalogr Clin Neurophysiol 85: 158-160.

Heatherton TF, Kozlowski LT, Frecker RC, Fagerstrom KO (1991). The Fagerstrom Test for nicotine dependence: a revision of the Fagerstrom Tolerance Questionnaire. Br J Addict 86: 1119-1127. Inghilleri M, Berardelli A, Marchetti P, Manfredi M (1996). Effects of diazepam, baclofen and thiopental on the silent period evoked by transcranial magnetic stimulation in humans. Exp Brain Res 109: 467-472.

Jacobsen LK, Krystal JH, Mencl WE, Westerveld M, Frost SJ, Pugh KR (2005). Effects of smoking and smoking abstinence on cognition in adolescent tobacco smokers. Biol Psychiatry 57: $56-66$.

Korchounov A, Ilic TV, Schwinge T, Ziemann U (2005). Modification of motor cortical excitability by an acetylcholinesterase inhibitor. Exp Brain Res 164: 399-405.

Kujirai T, Caramia MD, Rothwell JC, Day BL, Thompson PD, Ferbert A et al (1993). Corticocortical inhibition in human motor cortex. J Physiol 471: 501-519.

Kumari V, Gray JA, Ffytche DH, Mitterschiffthaler MT, Das M, Zachariah E et al (2003). Cognitive effects of nicotine in humans: an fMRI study. Neuroimage 19: 1002-1013.

Li SP, Park MS, Kim JH, Kim MO (2004). Chronic nicotine and smoke treatment modulate dopaminergic activities in ventral tegmental area and nucleus accumbens and the gammaaminobutyric acid type $B$ receptor expression of the rat prefrontal cortex. J Neurosci Res 78: 868-879.

Liepert J, Bar KJ, Meske U, Weiller C (2001a). Motor cortex disinhibition in Alzheimer's disease. Clin Neurophysiol 112: 1436-1441.

Liepert J, Schardt S, Weiller C (2001b). Orally administered atropine enhances motor cortex excitability: a transcranial magnetic stimulation study in human subjects. Neurosci Lett 300: 149-152.

Mansvelder HD, van Aerde KI, Couey JJ, Brussaard AB (2006). Nicotinic modulation of neuronal networks: from receptors to cognition. Psychopharmacology (Berl) 184: 292-305.

Oldfield RC (1971). The assessment and analysis of handedness: the Edinburgh Inventory. Neuropsychologia 9: 97-113.

Oliviero A, Leon AM, Holler I, Vila JF, Siebner HR, Della Marca G et al (2005). Reduced sensorimotor inhibition in the ipsilesional motor cortex in a patient with chronic stroke of the paramedian thalamus. Clin Neurophysiol 116: 2592-2598. 
Orth M, Amann B, Robertson MM, Rothwell JC (2005). Excitability of motor cortex inhibitory circuits in Tourette syndrome before and after single dose nicotine. Brain 128: 1292-1300.

Rothwell JC, Hallett M, Berardelli A, Eisen A, Rossini P, Paulus W (1999). Magnetic stimulation: motor evoked potentials. The International Federation of Clinical Neurophysiology. Electroencephalogr Clin Neurophysiol Suppl 52: 97-103.

Sacco KA, Termine A, Seyal A, Dudas MM, Vessicchio JC, Krishnan-Sarin S et al (2005). Effects of cigarette smoking on spatial working memory and attentional deficits in schizophrenia: involvement of nicotinic receptor mechanisms. Arch Gen Psychiatry 62: 649-659.

Sailer A, Molnar GF, Paradiso G, Gunraj CA, Lang AE, Chen R (2003). Short and long latency afferent inhibition in Parkinson's disease. Brain 126: 1883-1894.

Schwartz RD, Kellar KJ (1985). In vivo regulation of [3H]acetylcholine recognition sites in brain by nicotinic cholinergic drugs. J Neurochem 45: 427-433.

Siebner HR, Dressnandt J, Auer C, Conrad B (1998). Continuous intrathecal baclofen infusions induced a marked increase of the transcranially evoked silent period in a patient with generalized dystonia. Muscle Nerve 21: 1209-1212.

Thiel CM, Zilles K, Fink GR (2005). Nicotine modulates reorienting of visuospatial attention and neural activity in human parietal cortex. Neuropsychopharmacology 30: 810-820.
Tokimura H, Di Lazzaro V, Tokimura Y, Oliviero A, Profice P, Insola A et al (2000). Short latency inhibition of human hand motor cortex by somatosensory input from the hand. J Physiol 523(Part 2): 503-513.

Valls-Sole J, Pascual-Leone A, Wassermann EM, Hallett M (1992). Human motor evoked responses to paired transcranial magnetic stimuli. Electroencephalogr Clin Neurophysiol 85: 355-364.

Wang H, Sun X (2005). Desensitized nicotinic receptors in brain. Brain Res Brain Res Rev 48: 420-437.

Wassermann EM, Greenberg BD, Nguyen MB, Murphy DL (2001). Motor cortex excitability correlates with an anxiety-related personality trait. Biol Psychiatry 50: 377-382.

Yamazaki Y, Jia Y, Hamaue N, Sumikawa K (2005). Nicotineinduced switch in the nicotinic cholinergic mechanisms of facilitation of long-term potentiation induction. Eur J Neurosci 22: $845-860$.

Ziemann U (2003). Pharmacology of TMS. Suppl Clin Neurophysiol 56: $226-231$.

Ziemann U (2004). TMS and drugs. Clin Neurophysiol 115: 1717-1729.

Ziemann U, Lonnecker S, Steinhoff BJ, Paulus W (1996). Effects of antiepileptic drugs on motor cortex excitability in humans: a transcranial magnetic stimulation study. Ann Neurol 40: 367-378. 